\title{
Book Reviews-Livres Nouveaux
}

Transactions of the American Society for Artificial Internal Organs. Vol. XIV, 1968.

(George E. Schreiner Ed., Georgetown, University Hospital Washington D.C.). 1 vol. 470 p.; Prix $12 \$$.

Comme le precedent, ce volume a paru dans un délai record, moins de deux mois après la reunion de la Société, tenue à Philadelphie les 21 et 22 avril 1968. La variété, l'originalité, le caractère «simulant» des 78 communications presentees défient toute analyse sommaire. Le néphrologue voit avec intérêt que les méthodes de dialyse et la technologie des appareils, des shunts, des membranes constitue encore une partie importante du programme, témoignant de la vigueur des recherches dans ces domaines. La dialyse des poisons et des medicaments forme un chapitre special constamment mis à jour.J. Dormont Actualités Néphrologiques de l'Hôpital Necker 1968 (Ed. Médicales Flammarion, en langue française, Paris, 1968). 1 vol. 392 p.; 130 fig. Fr. 125.-. Les Actualités Néphrologiques proposent comme chaque année un inventaire des connaissances nouvelles dans le domaine du fonctionnement renal et dans celui du traitement des maladies rénales. La premiere partie de Touvrage est consacrée au rein artificiel, à la fois dans ses aspects les plus fondamentaux (choix des membra nes, circulation des fluides dans Tappareil) et dans certains problèmes precis que pose son usage; avantages respectifs des shunts externes et des fistules artérioveineuses, troubles du métabolisme calcique, troubles de Thémostase et intérêt du traitement anticoagulant au long cours. La deuxième partie de Touvrage est composée de 12 articles traítant de sujets varies: stenoses artérielles rénales, rénine, physiologie du segment grêle des néphrons, perméabilité glomérulaire aux grosses molecules, lipides dans les biopsies rénales, pyélonéphrites xanthogranulomateuses, effet renal de la thyrocalcitonine, hyperuricémie héréditaire précoce, histurie, de bit cardiaque dans Thypertension, néphroses osmotiques. Ces exposes precis et détaillés tout en restant sufi $1 / 8 a m m e n t$ didactiques, intéresseront tous les néphrologues. B. Varet 